\title{
Lattice Dynamic and Thermophysical Properties of AlSi (Silumine) Alloy: A DFT Study
}

\author{
R.K. Suthar ${ }^{1}$, N.Y. Pandya1,*, Adwait D. Mevada ${ }^{2}$, P.N. Gajjar ${ }^{3}$ \\ ${ }^{1}$ Parul University, P. O. Limda, Ta. Waghodia, Vadodara, 391760, Gujarat, India \\ ${ }^{2}$ President Science College, Ghatlodia, Ahmedabad, 380061, Gujarat, India \\ ${ }^{3}$ Department of Physics, University School of Sciences, Gujarat University, Ahmedabad, 380009, Gujarat, India
}

(Received 10 June 2020; revised manuscript received 12 February 2021; published online 25 February 2021)

\begin{abstract}
The present paper reports a comprehensive first-principles calculation of the structural, electronic and thermophysical properties of B1, B2 and B3 phases of AlSi (Silumine) alloy using plane-wave pseudopotential density functional theory (DFT). PAW type pseudopotential with the exchange correlation of Perdew-Burke-Ernzerhof (PBE) are used to compute properties of B1, B2 and B3 phases of AlSi alloy. Our computed equilibrium lattice constants are in excellent agreement with the reported results. To investigate the structural phase transitions beween different phases of AlSi alloy, volume dependence of energy and pressure dependence of enthaply are studied for B1, B2 and B3 phases of AlSi alloy. Further, the electronic band structure along with the total electronic density of states of B1, B2 and B3 phases of AlSi alloy are calculated at the ground states. Behaviour of the total electronic density of states of B1, B2 and B3 phases of AlSi is also studied with the increase in pressure up to $100 \mathrm{GPa}$. In addition, various finite temperature/pressure thermophysical properties such as the room temperature thermal equation of state, isothermal bulk modulus, coefficient of thermal expansion, heat capacity at constant volume and pressure, Debye temperature and Grüneisen parameter are computed for B1, B2 and B3 phases of AlSi alloy using quasi harmonic Debye model. Conclusions based on the structural, electronic and thermophysical properties of B1, B2 and B3 phases of AlSi alloy are summarized.
\end{abstract}

Keywords: AlSi, DFT, Negative pressure, Phonon dispersion curve, Electronic band structure, Thermophysical properties.

DOI: 10.21272/jnep.13(1).01013

PACS numbers: 71.18. + y, 71.15.Mb, 91.35.Ed

\section{INTRODUCTION}

Aluminum and its alloys are widely applied in the design and development of engineering structures where light weight is required [1]. In the high speed aerospace vehicles/automotive, it is required to reduce the weight of the total structure and improve the performance of the machine [2]. Silumine (AlSi) alloys are the most commonly used aluminum alloy in automobile and aerospace applications due to its excellent properties e.g. high strength, less ductility, lower thermal expansion rate, higher strength and low wear rate [3]. Some of the binary solid solutions in aluminium like $\mathrm{AlNi}, \mathrm{AlLi}, \mathrm{AlMg}$ and $\mathrm{AlSi}$ are studied by CALPHAD [4]. In this work [4], the crystallography of aluminium alloys is discussed. Structural and mechanical properties of AlSi alloys have been investgated by fast cooling of a levitated melt by Nikanorov et. al. [2]. The effect of Si content in AlSi alloy improved its mechanical and thermodynamic properties as compared to conventional $\mathrm{Al}$ alloys [3]. Doping of $\mathrm{Si}$ in $\mathrm{Al}$ reduces the thermal expansion coefficient, increases corrosion and wear resistance, and improves the casting and machining characteristics of the alloy [5]. With the increase in percentage of silicon in AlSi alloy, it becomes stronger and effective than pure $\mathrm{Al}$ [6]. The role of size and morphology of microstructural constituents (e.g SDAS, Si-particles and intermetalics) on mechanical properties of AlSi based casting alloy at room temperatures up to $500{ }^{\circ} \mathrm{C}$ have been investigated in detail by Zamani [7]. It is also studied that additions of minor alloying elements such as $\mathrm{Cu}$ and $\mathrm{Mg}$ also make better the mechanical properties and make the alloy responsive to heat treatment [7]. In addition, the effect of $\mathrm{Fe}, \mathrm{Ni}$ and $\mathrm{Cr}$ addition on the wear charcteristic of hyper-eutectic AlSi automotive alloy has been investigated [8]. Melt spinning process was introduced to study the rapid quenching process of Silumine with compositions in the range 0 at. \% to 33.9 at. \% Si from the melt at cooling rates between $10^{6}$ and $10^{7} \mathrm{~K} \cdot \mathrm{s}^{-1}$ [9]. Further the electronic properties and superconductivity of rapidly quenched $\mathrm{AlSi}$ alloys have been also investigated [10]. Impact of vibration on the solidification behaviour and tensile properties of an Al-18 wt. \% Si alloy have been studied to check the effect of vibration on the mechanical properties of castings [1]. In this test [1], tensile tests were implemented on specimens obtained with the different vibrating frequency levels. Vibration Characteristics of Hypo and Hyper Eutectic "AlSi" alloys related to machine and equipment have been also studied [11]. In order to predict the modal behaviour [11], the rigorous work is involved in modelling and analysis which has been done using

* niravbhai.pandya12730@paruluniversity.ac.in 
ANSYS workbench. An AlSi solid-state phase diagram is investigated using first-principles electronic structure calculations of formation enthalpies and quasiharmonic vibrational free energies by Ozolins et. al. [12]. They used the generalized-gradient approximation (GGA) and obtaid a good agreement with the experimental AlSi phase diagram [12]. Various structural, elastic and metal alloying properties of $\mathrm{Al}, \mathrm{Si}$ and binary $\mathrm{AlSi}$ are also reported by modified embedded atom method (MEAM) potentials [13]. Much research has been done on mechanical and casting properties of B2 phases of AlSi. Scarcity of electronic band structure, density of states and and thermophysical properties namely the room temperature thermal equation of state, isothermal bulk modulus, heat capacity at constant volume and pressure, Debye temperature and Grüneisen parameter has motivated us for the present investigations. In the present work, we describe ground state, electronic and thermophysical properties of B1-B2-B3 phases of AlSi alloy using plane wave pseudopotential density functional theory as implemented in Quantum ESPRESSO package [14]. Generally the solid crystallize in either the B1 $(\mathrm{NaCl}), \mathrm{B} 2(\mathrm{CsCl})$ or $\mathrm{B} 3(\mathrm{ZnS})$ structure. These solids undergo structural phase transition from B1-B2-B3 at positive or negative pressures [15]. The thermophysical properties are important characteristic to accurate and reliable design of melting and casting processes of alloys. The present work reports various finite temperature/pressure thermophysical properties such as the room temperature thermal equation of state, isothermal bulk modulus, coefficient of thermal expansion, heat capacity at constant volume and pressure, Debye temperature and Grüneisen parameter for B1, B2 and B3 phases of AlSi using Gibbs program [16]. Afte the introduction, the next section describes the computational methodology used in the present study. The section 3 describes the results and discussion. The section 4 describes the conclusions.

\section{COMPUTATIONAL METHODOLOGY}

As mentioned earlier, the present paper reports the ground state and lattice dynamical properties of B1, B2 and B3 phases of AlSi using plane wave pseudopotential density functional theory (DFT) as implemented in Quantum ESPRESSO code [14]. PAW pseudopotential with nonlinear core correction within generalized gradient approximation (GGA) was used to perform the presented calculation [17]. The $\mathrm{Al}$ and Si atoms occupy positions $(0,0,0)$ and $(0.50,0.50$, 0.50), respectively, in a Simple Cubic primitive cell. In the step of 0.1 a.u., lattice constants and the total energy calculations of $\mathrm{B} 1, \mathrm{~B} 2$ and $\mathrm{B} 3$ phases of $\mathrm{AlSi}$ were performed. The convergence test gave the kinetic energy cutoff of $100 \mathrm{Ry}, 110 \mathrm{Ry}$ and $110 \mathrm{Ry}$ for B1-AlSi, B2-AlSi and B3-AlSi, respectively. An $8 \times 8 \times 8$ Monkhorst-Pack (MP) k-point grid [18] for
Brilliouin-zone (BZ) sampling was used. This ensures the convergence of total energy to $0.1 \mathrm{mRy}$. Further the calculated total energies were fitted to Murnaghan equation of state [19], in order to estimate equilibrium lattice constant, isothermal bulk modulus and its pressure derivatives. At a given pressure, a structure is said to be stable one when it exhibits lowest enthalpy value [20,21]. The pressure stimulated structural phase transitions in AlSi is also investigated by calculating enthalpies of three cubic phases B1, B2 and B3 under the influence of positive and negative pressures. To study the electronic properties of $\mathrm{B} 1, \mathrm{~B} 2$ and $\mathrm{B} 3$ phases of AlSi, we present the electronic band structure along with total electronic density of states using the calculated equilibrium lattice constant. In the present work, we used quasi-harmonic Debye model implemented in pseudo code Gibbs [16] for the analysis of thermal effect. In this model [16], Debye temperature depends on the scaling function $f(\sigma)$. Hence it is required to introduce the value of the Poisson's ratios $\sigma$ in order to define $f(\sigma)$. We used 0.33 as a Poisson's ratio for B1, B2 and B3 phases of AlSi as reported in ref. [22]. After obtaining the equilibrium state for a given $(P, T)$, other thermophysical properties were evaluated by using the corresponding equilibrium volume in the appropriate expressions as given in ref. [16]. In the present work, we obtain the finite temperature/pressure thermophysical properties such as the isothermal bulk modulus, room temperature thermal equation of state, coefficient of thermal expansion, heat capacity at constant volume $(C \mathrm{~V})$ and at constant pressure $\left(C_{\mathrm{P}}\right)$, Debye temperature and Grüneisen parameter of B1-AlSi, B2-AlSi and B3-AlSi for computed cell parameters using the equations as mentioned in ref.[16].

\section{RESULTS AND DISCUSSION}

The obtained equilibrium lattice constant, isothermal bulk modulus and first order derivative of bulk modulus for B1-AlSi, B2-AlSi and B3-AlSi are shown below in Table 1 . Our calculated equilibrium lattice constant shows the variation of only $0.2 \%$ and $0.07 \%$ for $\mathrm{B} 1-\mathrm{AlSi}$ and $\mathrm{B} 2-\mathrm{AlSi}$, respectively, in comparision to the reported results [11,23] which shows good agreement of our results with the reported results [11,23]. In the absence of reliable literature data, we are not able to compare the calculated equilibrium lattice constant of B3-AlSi with other findings. To determine the structural parameters for the AlSi alloy, the total energy is calculated for three different phases $\mathrm{B} 1(\mathrm{NaCl}), \mathrm{B} 2$ $(\mathrm{CsCl})$ and $\mathrm{B} 3(\mathrm{ZnS})$. The total energy against volume curves for the three different phases are plotted in Fig. 1. The obtained energy of B2 phase is lower than B1 and B3 phases up to unit cell volume of around 250 (a.u.) ${ }^{3}$ which predicts the structural stability of the B2 phase of AlSi [24] in comparison to B1 and B3 phases. For higher volume than this, the structural 
phase transition of the phase B2 to B3 is seen for AlSi. Further, to check the structural phase transition, we have calculated the pressure dependence of enthalpies for B1-AlSi, B2-AlSi and B3-AlSi under the positive and negative pressure; and the corresponding results are shown in Fig. 2. It is observed that no structural phase transitions take place in B1, B2 and B3 phases of AlSi up to $1000 \mathrm{GPa}$ and remain stable at such a high pressure. As observed in Fig. 2, phase transition is predicted at $-3.57 \mathrm{GPa}$ negative pressure from $\mathrm{B} 1(\mathrm{NaCl})$ to $\mathrm{B} 3$ $(\mathrm{ZnS})$ and $\mathrm{B} 2 \quad(\mathrm{CsCl})$ to $\mathrm{B} 3 \quad(\mathrm{ZnS})$ at $-2.88 \mathrm{GPa}$.

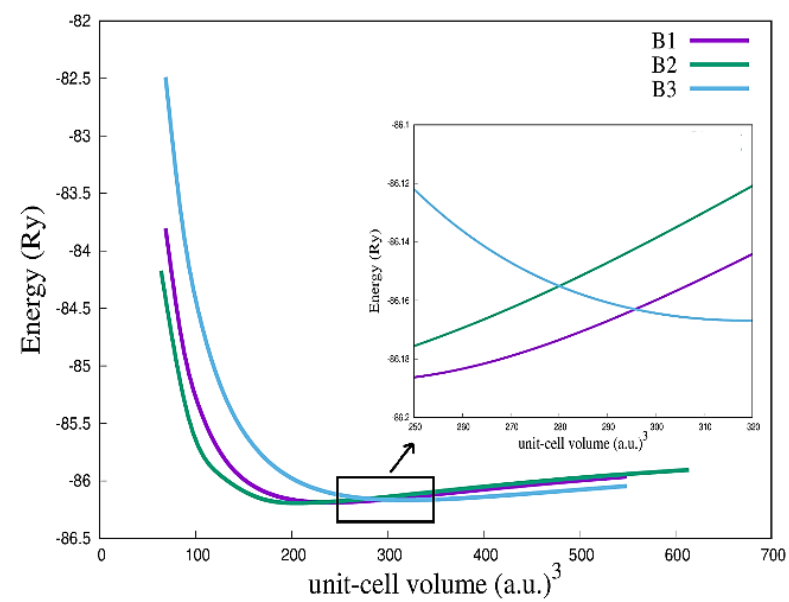

Fig. 1 - Volume dependence of energy of B1, B2 and B3 phases of AlSi

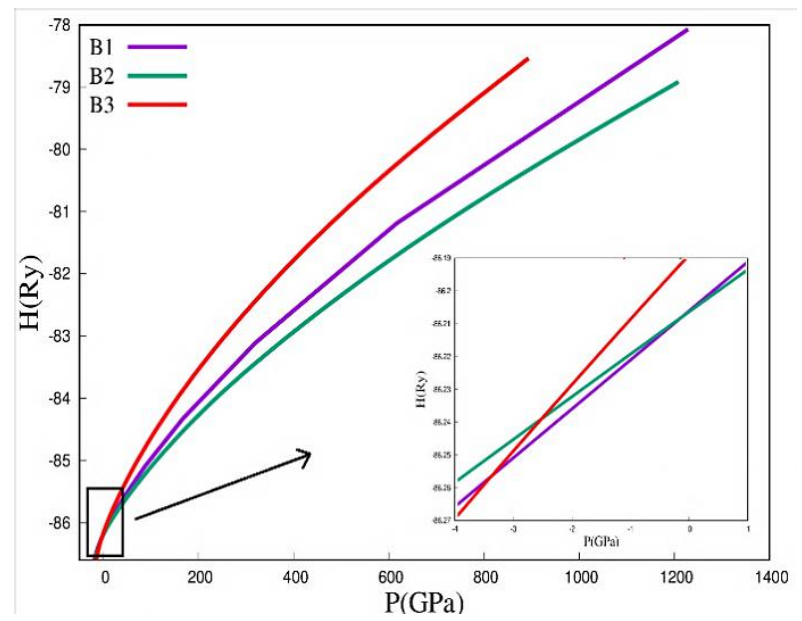

Fig. 2 - Enthalpy versus pressure curve for B1, B2 and B3 phases of AlSi

The calculated electronic band structure of B1-AlSi, B2-AlSi and B3-AlSi along the high symmetry directions in first Brillion zone are shown in Fig. 3, Fig. 4 and Fig. 5, respectively. The computed Fermi energies for B1-AlSi, B2-AlSi, and B3-AlSi are $7.06 \mathrm{eV}, 9.03 \mathrm{eV}$ and $3.74 \mathrm{eV}$, respectively. Crossig of the energy bands

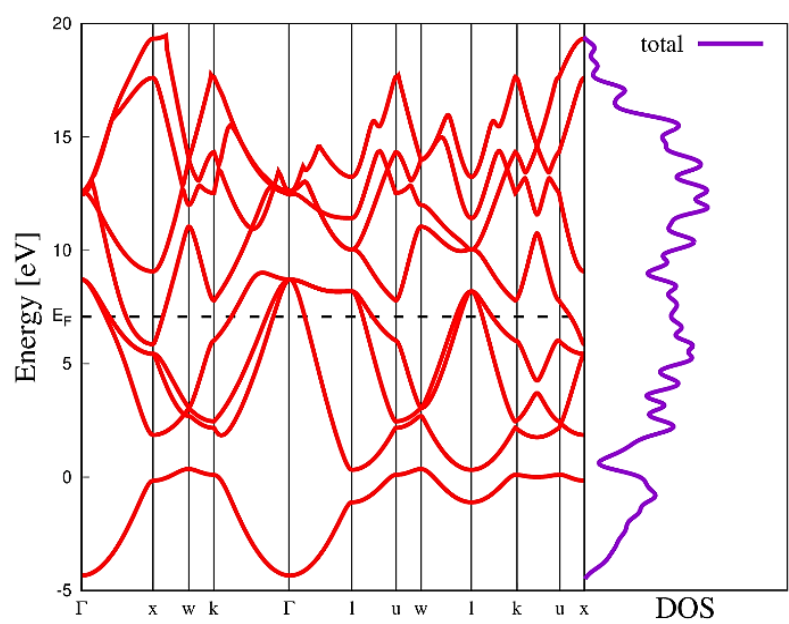

Fig. 3 - Electronic band structure and total electronic density of states (TDOS) of B1 phase of AlSi

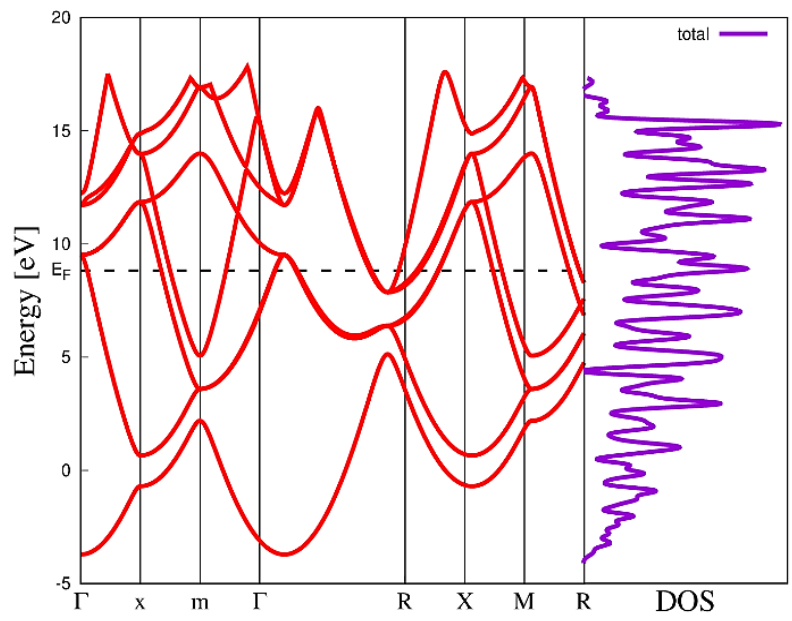

Fig. 4 - Electronic band structure and total electronic density of states (TDOS) of $\mathrm{B} 2$ phase of AlSi

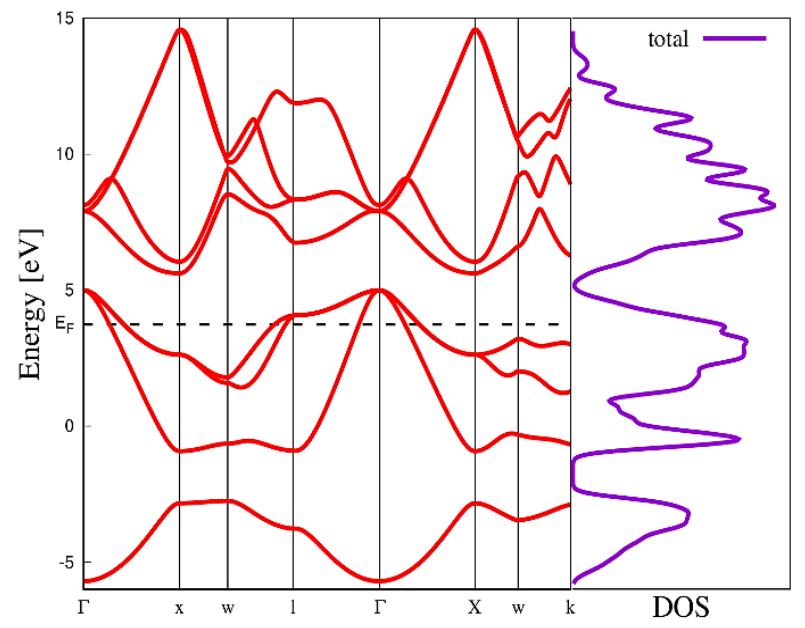

Fig. 5 - Electronic band structure and total electronic density of states (TDOS) of B3 phase of AlSi 
at the Fermi level $E_{\mathrm{F}}$ in the band structure of B1 and B2 phases of AlSi confirms the metallic behavior of $\mathrm{B} 1$ and $\mathrm{B} 2$ phases of AlSi. The dispersive nature of electronic bands predict the high electron mobility in B1 and B2 phases of AlSi. As it is seen from Fig. 5 that the electronic states in valance band crosses the Fermi energy level with presence of energy gap between conduction band and valance band in B3AlSi. This predicts the semi metal nature of B3-AlSi. Total electronic density of states of B1, B2 and B3 phase of AlSi resemble all the features of electronic bandstructure. In the literature, we found electronic band structure of B2 phase and our computed electronic band structure of B2-AlSi shows good agreement [23]. Due to nonavailability of literature on electronic band structure of B1-AlSi and B3-AlSi, we are not able to compare our computed electronic band structure of B1-AlSi and B3-AlSi with the other findings. We also investigated pressure dependence of energy bands of B1-AlSi, B2-AlSi and B3-AlSi which are shown in Fig. 6, Fig. 7 and Fig. 8, respectively. For this TDOS are computed at 0,50 , and $100 \mathrm{GPa}$. It is seen that electronic bands shift towards higher energy sides with the increase in pressure which implies that electrons occupy higher energy states with the increase in pressure. The Fermi level shifts towards higher energy from $E_{\mathrm{F}}(0 \mathrm{GPa})=7.13 \mathrm{eV}$ to $E_{\mathrm{F}}(50 \mathrm{GPa})=8.57 \mathrm{eV}$ to $E_{\mathrm{F}}(100 \mathrm{GPa})=9.39 \mathrm{eV}$ for $\mathrm{B} 1-\mathrm{AlSi}$. The similar shifting of $\mathrm{E}_{\mathrm{F}}$ with pressure also observe for B2-AlSi and B3-AlSi.

Table 1 - Calculated structural properties of B1, B2 and B3 phases of AlSi alloy

\begin{tabular}{|c|c|l|c|l|c|c|}
\hline \multirow{2}{*}{$\begin{array}{l}\text { Proper- } \\
\text { ties }\end{array}$} & \multicolumn{2}{|c|}{ B1 } & \multicolumn{2}{c|}{ B2 } & \multicolumn{2}{c|}{ B3 } \\
\cline { 2 - 7 } & Present & Other & Present & Other & Present & Other \\
\hline$a_{0}(\AA)$ & 5.2192 & $\begin{array}{l}5.2317 \\
{[11],} \\
5.2414 \\
{[11]}\end{array}$ & 3.1541 & $\begin{array}{l}3.1518 \\
{[23],}\end{array}$ & 5.7378 & - \\
& & & $\begin{array}{l}\text { 3.1688 } \\
{[11],} \\
3.1912 \\
{[11]}\end{array}$ & & \\
\hline $\begin{array}{c}B_{0} \\
(\mathrm{GPa})\end{array}$ & 62.8 & - & 59.9 & - & 48.0 & - \\
\hline$B_{0}{ }^{\prime}$ & 3.63 & - & 3.27 & - & 3.31 & - \\
\hline
\end{tabular}

In the present study, we have also computed the thermophysical properties of B1-AlSi, B2-AlSi and B3-AlSi with the help of GIBBS program [16].

The normalized volume-pressure diagram of B1, B2 and B3 phases of AlSi at room temperatures show that the unit cell volume decreases smoothly and no abrupt change occurs with the increase in pressure up to $40 \mathrm{GPa}$. This further reveal that the crystal structure of the B1, B2 and B3 phases of AlSi are stable up to a pressure of $40 \mathrm{GPa}$ [25].
The bulk modulus is nearly a constant when $T<100 \mathrm{~K}$. However, it decreases linearly with the increase in temperature $T$, when $T>100 \mathrm{~K}$. The Bulk modulus decreases $9.67 \%, 0.05 \%$ and $7.14 \%$ for B1, B2 and B3 phases, respectively, as temperature increases from 0 to $1073 \mathrm{~K}$. This can be linked with the thermal expansion of the material [25]. It can be seen that $\alpha$ increases rapidly with the increase in temperature at low temperature region up to $400 \mathrm{~K}$ and then increases smoothly at high temperatures. Room temperature $(300 \mathrm{~K})$ thermal expansion coeffiecient for B1-AlSi, B2-AlSi and B3-AlSi are $10.29 \times 10^{-6} / \mathrm{K}, \quad 0.95 \times 10^{-6} / \mathrm{K}$ and $8.02 \times 10^{-6} / \mathrm{K}$, respectively.

The computed values of constant-volume specific heats $C_{V}$ and constant-pressure specific heat $C_{P}$ of B1-AlSi, B2-AlSi and B3-AlSi are shown in Fig. 9.

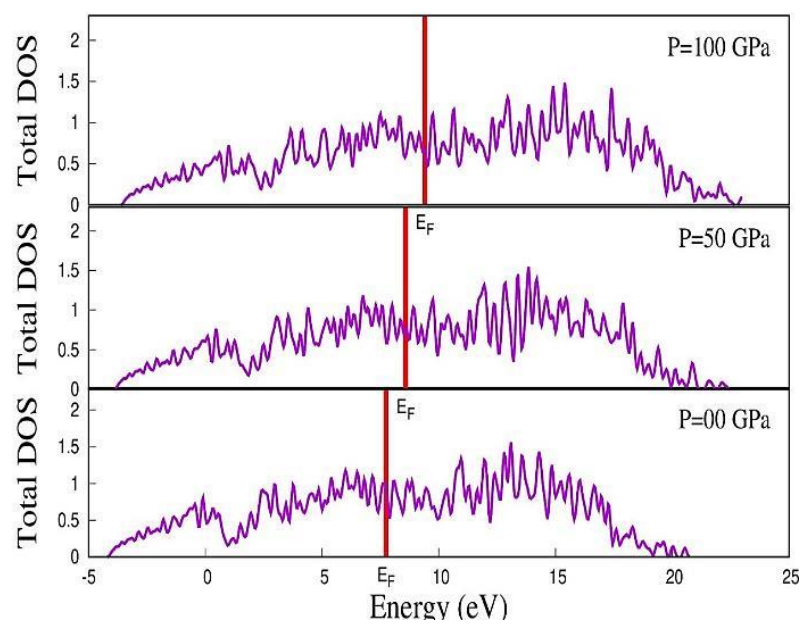

Fig. 6 - Presuure dependence of total electronic DOS (TDOS) of $\mathrm{B} 1$ phase of $\mathrm{AlSi}$

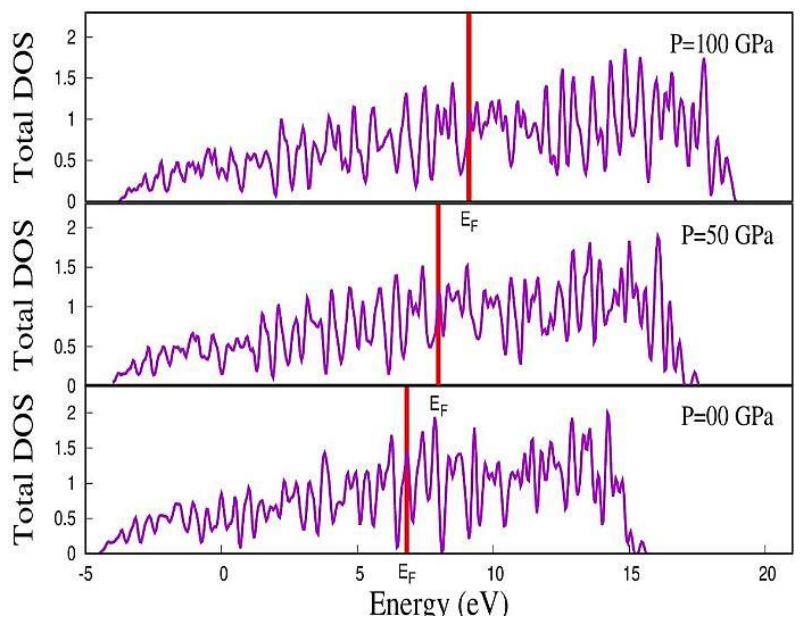

Fig. 7 - Presuure dependence of total electronic DOS (TDOS) of $\mathrm{B} 2$ phase of $\mathrm{AlSi}$ 


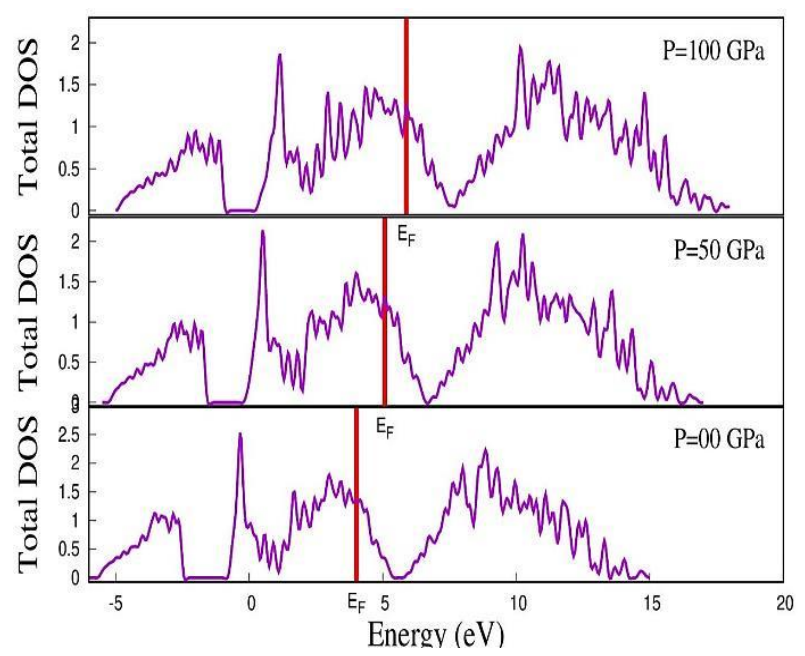

Fig. 8 - Presuure dependence of total electronic DOS (TDOS) of B3 phase of AlSi

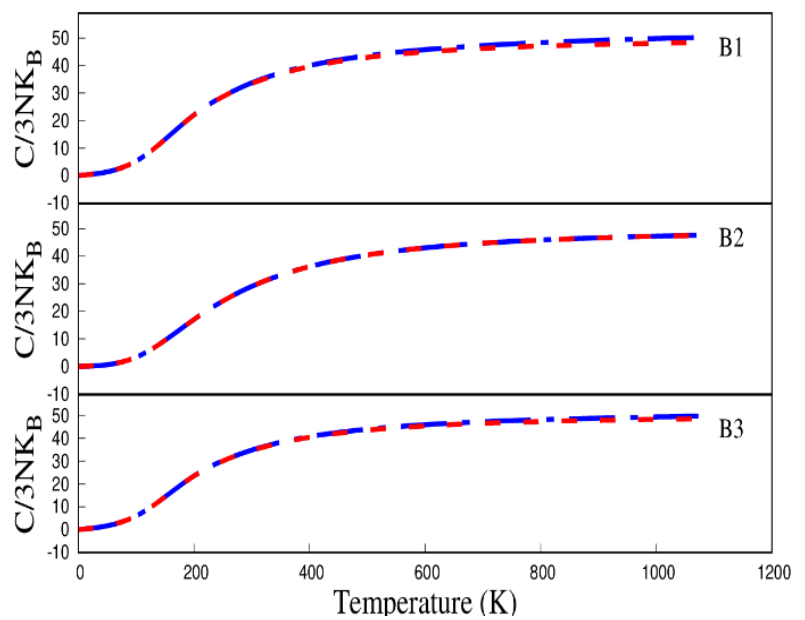

Fig. 9 - Specific heats as a function of temperature for B1, $\mathrm{B} 2$ and B3 phases of AlSi

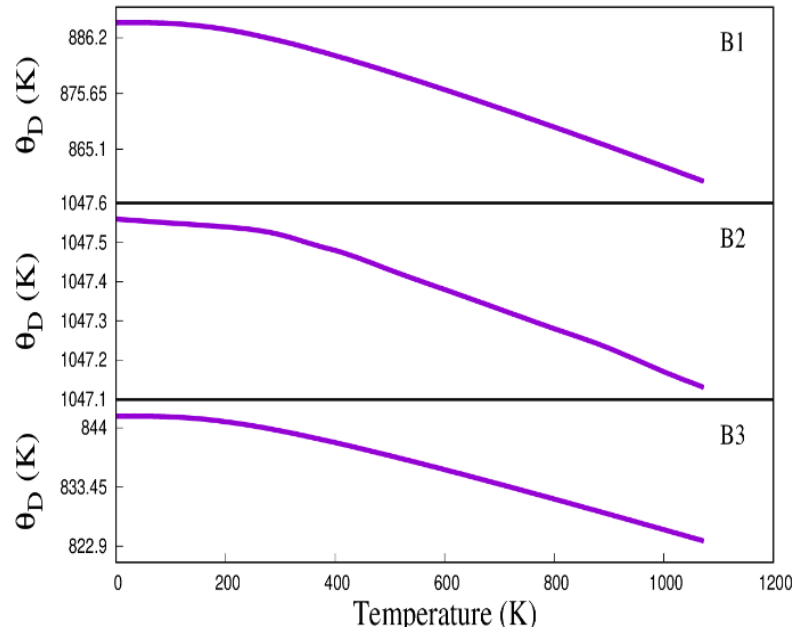

Fig. 10 - Temperature dependence of Debye temperature for $\mathrm{B} 1, \mathrm{~B} 2$ and B3 phases of AlSi

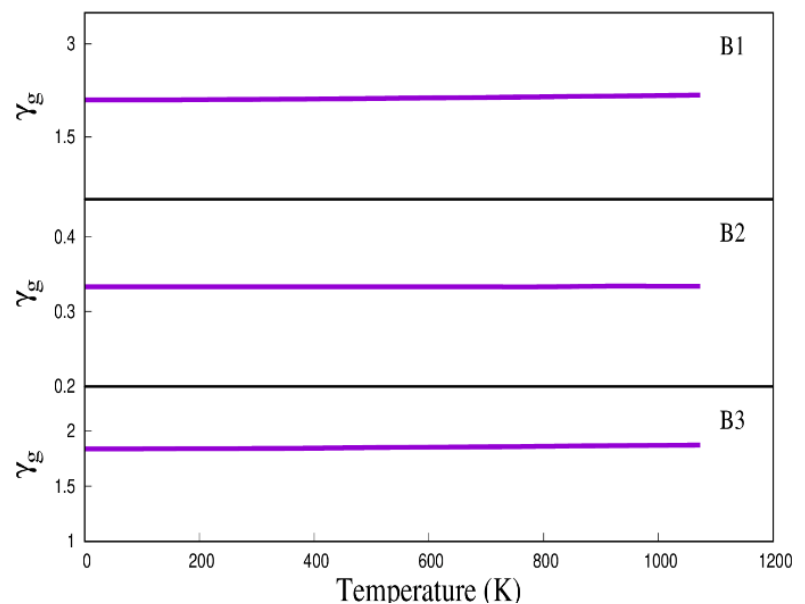

Fig. 11 - Grüneisen parameter as a function of temperature for $\mathrm{B} 1, \mathrm{~B} 2$ and $\mathrm{B} 3$ phases of $\mathrm{AlSi}$

It is seen that $C_{P}$ values are slightly higher (and hence overlapping) than $C_{V}$. Values of $C_{P}$ are slightly higher than values of $\mathrm{C}_{V}$,which can be explained by the relation between $C_{P}$ and $C_{V}$ as $C_{P}-C_{V}=$ $\left(a^{2} \cdot B V T\right)$; with $\alpha_{v}, B, V$ and $T$ are the coefficient of volume thermal expansion, bulk modulus, volume and absolute temperature, respectively [25].

Debye temperature $\left(\theta_{\mathrm{D}}\right)$ is one of the most important integral parameters of crystals in the solid state physics, which is associated with the natural frequency of the lattice vibrations of solids material and it also defines its stability. As per thumb rule, a higher Debye temperature implies a higher associated thermal conductivity. Fig. 10 displays the temperature dependence of Debye temperature at pressure $P=0$ for B1-AlSi, B2-AlSi and B3-AlSi. It can be seen that $\theta_{\mathrm{D}}$ remains nearly constant till $T<200 \mathrm{~K}$ and decreases linearly at $T>200 \mathrm{~K}$. Debye temperature $\theta_{\mathrm{D}}$ can be used to measure the stiffness of solids because vibration frequency is proportional to the square root of the stiffness within the harmonic approximation [26]. Thus, the plot given in Fig. 10 also indicates the variation of stiffness of B1, B2 and B3 phases of AlSi as a function of temperature.

In Fig. 11, the computed Grüneisen parameters are plotted for the different temperature values at pressure $P=0$ for $\mathrm{B} 1, \mathrm{~B} 2$ and $\mathrm{B} 3$ phases of AlSi. Grüneisen parameter varies from 2.095 to 2.173 for B1-AlSi, 0.333 to 0.334 for B2-AlSi and 1.838 to 1.873 for B3-AlSi with the temperature variation from $0 \mathrm{~K}$ to $1073 \mathrm{~K}$. A little change in $\gamma_{\mathrm{g}}$ with temperature agrees well with Grüneisen's assumption [25].

\section{CONCLUSIONS}

In the present work, we have studied mainly the structural, electronic and thermophysical properties of B1, B2 and B3 phases of AlSi (Silumine) alloy. The calculated equilibrium lattice constants of B1, B2 and B3 phases of AlSi are in accordance with the other 
reported data. The energy-volume relation predicts the structural stability of B2-AlSi compared to B1 and B3 phases of AlSi alloy up to a unit cell volume of 250 (a.u.) ${ }^{3}$ and aftwerward predicts the possibility of the structural phase transition between B1, B2 and B3 phases. The pressure-induced structural phase transition between B1-, B2- and B3-AlSi suggests that this phase transition occurs at compression pressures. From the electronic band structure and the total electronic density of states (TDOS) of B1 and B2 structures of $\mathrm{AlSi}$, we noticed a metallic nature with high electron mobility, whereas B3-AlSi structure shows a semimetal nature. Pressure dependence of TDOS predicts that electronic energy bands and the Fermi level $E_{F}$ shift towards higher energy sides with an increase in pressure up to $100 \mathrm{GPa}$ for $\mathrm{B} 1, \mathrm{~B} 2$ and B3 phases of AlSi (Silumine) alloy.

Further from the computed thermophysical properties, the following results can be concluded. Volume-pressure diagram of the B1, B2 and B3 structures of AlSi concludes the stable structure up to $40 \mathrm{GPa}$ pressure, and no abrupt change in volume up to this pressure. The bulk modulus is almost constant for all three phases, when $T<100 \mathrm{~K}$. However, it decreases linearly with the increase in temperature

\section{REFERENCES}

1. G. Chirita, I. Stefanescu, D. Soares, F.S. Silva, Mater. Des. 30, 1575 (2009).

2. S.P. Nikanorov, M.P. Volkov, V.N. Guri, L.I. Derkachenko, Mater. Sci. Eng. A 390, 63 (2005).

3. Akyüz, ASTRJ 10, 51 (2016).

4. P.N.H. Nakashima, The Crystallography of Aluminum and Its Alloys, in Encyclopedia of Aluminum and Its Alloys (Ed. G.E. Totten, M. Tiryakioğlu, O. Kessler), 488 (Boca Raton: CRC Press: 2018).

5. V. Sklyarchuk, 'Yu. Plevachuk, A. Yakymovych, S. Eckert, G. Gerbeth, K. Eigenfeld, Int. J. Thermophys. 30, 1400 (2009).

6. A. Sathya Prakash, M.S. Hithesh, B. Vishnuprasad, K.S. Umashankar, National Conference on Advances in Mechanical Engeineerinh Science 3, 365 (2016).

7. AlSi Cast Alloys -Microstructure and Mechanical Properties at Ambient and Elevated Temperature. Mohammadreza Zamani. School of Engineering Jönköping University Licentiate thesis, Dissertation Series No 7, (2015).

8. M.S. Kaisera, S.H. Sabbirb, M.S. Kabirb, M.R. Soummob, M. Al Nurb, Mater. Res. 21, e20171096 (2018).

9. A. Bendijk, R. Delhez, L. Katgerman, Th.H. De Keijser, E.J. Mittemeijer, N.M. Van der Pers, J. Mat. Sci. 15, 2803 (1980).

10. J. Chevrier, D. Pavuna, F. Cyrot-Lackmann, Phys. Rev. B 36, 9115 (1987).

11. V. Somashekar, IJERT 3, 3080 (2014).
$T$, when $T>100 \mathrm{~K}$. It can be concluded that $\alpha$ increases rapidly with the increase in temperature in the low-temperature region up to $400 \mathrm{~K}$ and then increases smoothly at high temperatures. It is observed that both $C_{P}$ values are negligibly more for all three phases, when $T<500 \mathrm{~K}$, and notably more for B1 and B3 phases above $T>500 \mathrm{~K}$. Variation of stiffness of B1, B2 and B3 phases of AlSi (Silumine) alloy with temperature can be estimated by studying the temperature dependence of $\theta_{\mathrm{D}}$. An extermely small change in $\gamma_{\mathrm{g}}$ with temperature agrees well with Grüneisen's assumption.

\section{ACKNOWLEDGEMENTS}

PNG acknowledged the computer facility developed under DST-FIST Level-I (No. SR/FST/PSI-097/2006 dated 20th December 2006 and No. SR/FST/PSI198/2014 dated 21st November 2014) programmes of the Department of Science and Technology, the Government of India, New Delhi, India, and support under DRS-SAP-I (No. F-530/10/DRS/2010 (SAP-I) dated November 2010 and No. F.530/17/DRS-II/2018 (SAP-I) dated 17th April 2018) of the University Grants Commission, New Delhi, India, is highly.

12. V. Ozolins, B. Sadigh, M. Asta, J. Phys.: Condens. Matter 17, 2197 (2005).

13. B. Jelinek, S. Groh, M.F. Horstemeyer, Phys. Rev. B 85, 245102 (2012).

14. www.quantum-espresso.org

15. P. Bhardwaj, S. Singh, Cent. Eur. J. Chem. 10, 1391 (2012).

16. M.A. Blanco, E. Francisco, V. Luana, Comp. Phys. Commun. 158, 57 (2004).

17. Y. Zhao, N.E. Schultz, D.G. Truhlar, J. Chem. Phys. 123, 161103 (2005).

18. B. Schoeters, O. Leenaerts, G. Pourtois, B. Partoens, J. Appl. Phys. 118, 104306 (2015).

19. N.Y. Pandya, A.D. Mevada, P.N. Gajjar, Comput. Mater. Sci. 123, 287 (2016).

20. C.U.M. Trinadh, Ph.D. thesis, Anna University, Chennai (India) (1997).

21. M. Fang, S. Tang, Z. Li, X. Wang, Comput. Mech. 50, 645 (2012).

22. F. Lasagni, H. Peter Degischer, J. Compos. Mater. 44 (2010).

23. A. Jain, S.P. Ong, G. Hautier, W. Chen, W.D. Richards, S. Dacek, S. Cholia, D. Gunter, D. Skinner, G. Ceder, K.A. Persson, APL Mater. 1, 011002 (2013).

24. N. Munjal, M.C. Mishra, G. Sharma, B.K. Sharma, J. Theor. Chem. 2013, 349870 (2013).

25. N.Y. Pandya, A.D. Mevada, P.N. Gajjar, Adv. Sci. Lett. 22, 3738 (2016).

26. T. Yokoyama, e-J. Surf. Sci. Nanotech. 10, 486 (2012). 